\title{
Table of Contents Use
}

\section{The Mechanism of Isotactic Polypropylene Formation with $C_{1}$-symmetric Metallocene Catalysts}

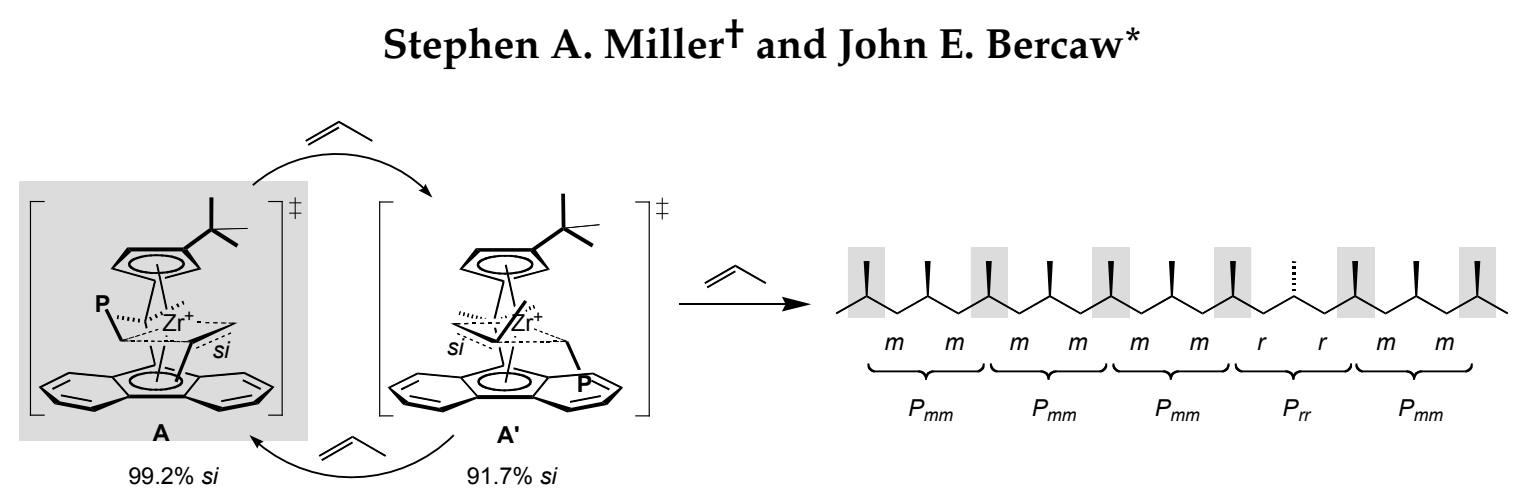

A variety of zirconocenes related to $C_{1}$-symmetric catalyst $\mathbf{A}$ have been investigated in the MAO-cocatalyzed isoselective polymerization of propylene. Examination of the polypropylene microstructures generated by $\mathbf{A}$ and these modified zirconocene catalysts under various reaction conditions indicates that an alternating mechanism is operative in which both sites of the metallocene are generally employed for monomer insertion. 